\title{
Bioinformatics analysis of biomarkers of aristolochic acid-induced early nephrotoxicity in embryonic stem cells
}

\author{
LI WANG $^{1 *}$, SHANSHAN MAN $^{1 *}$ and YUHONG BIAN ${ }^{2}$ \\ ${ }^{1}$ Pharmaceutical Sector, Tianjin Second People's Hospital, Tianjin Institute of Liver Disease, Tianjin 300192; \\ ${ }^{2}$ School of Integrative Medicine, Tianjin University of Traditional Chinese Medicine, Tianjin 301617, P.R. China
}

Received May 6, 2020; Accepted January 25, 2021

DOI: $10.3892 /$ etm.2021.9939

\begin{abstract}
The present study aimed to identify key genes as potential biomarkers for early nephrotoxicity induced by aristolochic acid (AA) in embryonic stem cells (ESCs). An MTT assay was performed to determine the cytotoxicity of AA in ESCs. Differentially expressed genes (DEGs) were identified using the DNA-Chip Analyzer following microarray analysis. Gene Ontology analysis was performed to determine functional terms enriched by the DEGs in the categories biological process, cellular component and molecular function. Furthermore, the DEGs were subjected to Kyoto Encyclopedia of Genes and Genomes analysis to determine pathways they were accumulated in. Furthermore, a protein-protein interaction network was constructed using Cytoscape 3.2 software. Tumor protein 53 apoptosis effector (Perp), cation transport regulator-like 1 (Chac1), adrenoceptor $\beta 2$ and Wnt6 were selected for confirmation by reverse transcription-quantitative (RT-q) PCR analysis. A total of 72 DEGs (49 upregulated and 23 downregulated) were identified. The DEGs were enriched in functional terms and pathways associated with nephrotoxicity and participated in 92 pathways. A total of two hub genes, fructose-1,6-bisphosphatase (Fbp)1 and Fbp2, were filtered out from the interaction network. Perp and phorbol-12-myristate-13-acetate-induced protein 1 were demonstrated to have vital roles in the p53 signaling pathway which was indicated in the interaction network. The results of the RT-qPCR analysis were consistent with the microarray data. Taken together, the present study suggested that hub genes involved in the p53 pathway, including Fbp1, Fbp2 and Perp, may serve as potential biomarkers for early nephrotoxicity induced by AA.
\end{abstract}

Correspondence to: Professor Yuhong Bian, School of Integrative Medicine, Tianjin University of Traditional Chinese Medicine, 10 Poyanghu Road, Tuanbo New Town, Jinghai, Tianjin 301617, P.R. China

E-mail: yuhongbiantj@163.com

*Contributed equally

Key words: aristolochic acid, nephrotoxicity, genomics, analysis

\section{Introduction}

Aristolochic acid (AA) is a major component of several Chinese herbs that exhibits a wide range of pharmacological effects, including anti-infective, anticancer and immunostimulatory effects, and may be used for termination of pregnancy $(1,2)$. Clinical reports and experimental studies have demonstrated that AA causes renal toxicity (3), acute renal failure (ARF) (4) and interstitial fibrosis (5). AA nephropathy (AAN) has become a worldwide problem (6). Recently, the use of AA-containing drugs was pronounced forbidden in the US, Canada, several countries in Europe and even certain countries in Asia.

Pathological analysis demonstrated that the infiltration of monocytes/macrophages and $\mathrm{T}$ lymphocytes was present in the necrotic area of proximal renal tubules in a rat model of AAN (7). However, the molecular mechanisms underlying the nephrotoxicity of AA have remained to be fully elucidated. Previous studies have reported that human renal toxicity attributes to the mutagenicity and DNA adducts derived by AA in the kidney and other tissue types (8). This suggests that AA-induced genetic alterations may have an important role in renal toxicity induced by AA. In addition, changes in mRNA expression are considered one of the earliest events prior to the occurrence of clinical symptoms. Microarray technology makes it possible to study genome-wide expression profiles and determine the potential molecular mechanisms of Chinese medicine with complex components. Previous studies have aimed to determine more accurate and earlier toxicity biomarkers for clinical and preclinical safety assessment using genomics analysis $(9,10)$. Thus, there remains an urgent requirement to perform genomics analysis to identify accurate biomarkers of AA-induced renal toxicity, which may contribute to the clinical drug safety of AA.

Terminal differentiation cells, such as renal tubular epithelial cells, are commonly used for nephrotoxic tests in vitro. These cells are primary cells or established cell lines; however, they cannot function as specialized organs or the whole body during long-term cultivation. Embryonic stem cells (ESCs) are pluripotent cells isolated from early embryos (11), which have highly undifferentiated potential and are capable of differentiating into all kinds of body tissues and organs, including liver, kidney, heart and nerves (12). It has been reported that ESCs are sensitive to drug stimulation and may thus serve as important tools for in vitro assessment of drug 
toxicity (13). The application of ESCs in toxicology studies will help to overcome the disadvantages of time consumption and low sensitivity of in vivo studies and also overcome the disadvantages of using terminal cells that cannot accurately represent the target organ.

The present study aimed to identify accurate biomarkers of AA-induced renal toxicity on ESCs. Genomics analysis was performed to screen for genes with changes in expression levels in ESCs following treatment with AA in order to determine the potential biological processes through which AA induces renal toxicity.

\section{Materials and methods}

Animals. A total of 50 Kunming mice (age, 6 weeks; body weight, $20 \pm 2 \mathrm{~g}$ ), half of them were male and half female, were obtained from Charles River Laboratory Animal Co., Ltd. The animals were fed with normal feed and water ad libitum and kept at $25 \pm 2^{\circ} \mathrm{C}$ with a humidity of $60 \%$. Animals were acclimated for one week and then female and male mice were housed in pairs within one cage (14). On day 12.5 after pregnancy was determined in the female mice through observing the presence of vaginal suppositories, animals were anesthetized by intraperitoneal injection of $10 \%$ chloral hydrate $(300 \mathrm{mg} / \mathrm{kg})$ and then sacrificed by neck dislocation. The experimental protocol conformed to the National Institutes of Health (NIH) Guide for the Care and Use of Laboratory Animals.

Cell culture. Mouse embryonic fibroblasts (MEFs) were obtained from the embryos of Kunming mice (15) at 12.5 days of gestation and maintained in high-glucose DMEM (4.5 g glucose/1; Gibco; Thermo Fisher Scientific, Inc.) supplemented with $10 \% \mathrm{FBS}, 50 \mathrm{U} / \mathrm{ml}$ penicillin and $50 \mathrm{mg} / \mathrm{ml}$ streptomycin and $1 \%$ non-essential amino acids (Gibco; Thermo Fisher Scientific, Inc.). MEFs were treated with $10 \mathrm{mg} / 1$ mitomycine for $2.5 \mathrm{~h}$ to attain feeder cells. The murine (m)ESCs (CRL-11632 $2^{\mathrm{TM}}$ ) were obtained from Shanghai Institutes for Biological Sciences, Chinese Academy of Sciences and maintained in DMEM ( $4.5 \mathrm{~g}$ glucose/1; Gibco; Thermo Fisher Scientific, Inc.) supplemented with 15\% FBS, $2 \mathrm{mM}$ glutamine (Sigma Aldrich; Merck KGaA), $50 \mathrm{U} / \mathrm{ml}$ penicillin, $50 \mathrm{mg} / \mathrm{ml}$ streptomycin (Sigma Aldrich; Merck $\mathrm{KGaA}$ ), $1 \%$ non-essential amino acids (Gibco; Thermo Fisher Scientific, Inc.), $0.1 \mathrm{~mm} \beta$-mercaptoethanol (Sigma Aldrich; Merck KGaA) and leukemia inhibitory factor $(1,000 \mathrm{U} / \mathrm{ml}$; Gibco; Thermo Fisher Scientific, Inc.) on MEF feeders to undifferentiate them at $37^{\circ} \mathrm{C}$ in an atmosphere containing 5\% $\mathrm{CO}_{2}$. The present study was approved by the Animal Ethics Committee of Tianjin University of Traditional Chinese Medicine (Tianjin, China).

MTT assay. An MTT assay was performed to determine the cytotoxicity of AA. ESCs were seeded into 96-well plates at a density of $1.5 \times 10^{5} / \mathrm{ml}(0.1 \mathrm{ml})$ per well. Different concentrations of AA $(50.0,25.0,12.5,10.0,6.25,5.0,3.13$ and $2.5 \mu \mathrm{g} / \mathrm{ml}$; Tianjin Yifang Science \& Technology Co., Ltd.) were added to each well, followed by incubation at $37^{\circ} \mathrm{C}$ in an atmosphere with $5 \% \mathrm{CO}_{2}$ for $48 \mathrm{~h}$. The supernatant was discarded and $20 \mu 1$ of MTT $(0.5 \mathrm{mg} / \mathrm{ml}$; Sigma-Aldrich Merck KGaA) was added to each well, followed by incubation for $4 \mathrm{~h}$ at $37^{\circ} \mathrm{C}$ in an atmosphere with 5\% $\mathrm{CO}_{2}$. The supernatant was discarded, the purple formazan crystals that had formed were dissolved using DMSO $(150 \mu \mathrm{l})$ and cytotoxicity was subsequently measured at a wavelength of $570 \mathrm{~nm}$. The concentration that reduced the number of viable cells by $10 \%\left(\mathrm{IC}_{10}\right)$ of $\mathrm{AA}$ was calculated using SPSS software (version 11.5; SPSS, Inc.) and then AA was used at this concentration in the following experiments. There were 3 independent replicates and 6 multiple wells per experiment.

Drug concentrations and treatment regime for microarray. ESCs were seeded into 6 -well plates at a density of $1.5 \times 10^{5} / \mathrm{ml}(2 \mathrm{ml})$ per well and incubated at $37^{\circ} \mathrm{C}$ in an atmosphere with $5 \% \mathrm{CO}_{2}$. Vitamin $\mathrm{C}(\mathrm{Vc})$ was used as a non-toxic control (16). Cells were subsequently treated with AA $\left(\mathrm{IC}_{10}\right)$ or Vc $(7.92 \mu \mathrm{g} / \mathrm{ml})$ and cultured for $48 \mathrm{~h}$; these conditions were selected from the MTT assay and other preliminary experiments (data not shown).

Microarray assay. RNA was extracted from cells following exposure to AA or Vc for $48 \mathrm{~h}$. In brief, following drug treatment, cells were collected and extracted using TRIzol ${ }^{\circledR}$ reagent (Invitrogen; Thermo Fisher Scientific, Inc.) at $-80^{\circ} \mathrm{C}$. Total RNA was purified using the RNeasy ${ }^{\circledR}$ Mini kit (Qiagen China Co., Ltd.), with the additional DNase treatment (RNase-Free DNase Set; Qiagen China Co., Ltd.), according to the manufacturer's protocols. Total RNA was quantified using the NanoDrop 1000 spectrophotometer (NanoDrop Technologies; Thermo Fisher Scientific, Inc.), by determining the optical density at $260 \mathrm{~nm}\left(\mathrm{OD}_{260}\right) / \mathrm{OD}_{280}$ ratio. Total RNA was subsequently purified using the Qiagen RNeasy total RNA cleanup kit (Qiagen China Co., Ltd.) and the concentration was measured using a NanoDrop 1000.

The microarray experiment was performed using the GeneChip $^{\circledR}$ 3IVT Express kit (Affymetrix; Thermo Fisher Scientific, Inc.), according to the manufacturer's protocol. For the microarray data analysis, 6 samples in total (including 3 control and 3 AA-treated samples) were included. For each sample, $100 \mathrm{ng}$ of purified RNA was reverse transcribed using T7-(dT) 24 primers containing a T7 RNA polymerase promoter to generate first-strand complementary (c)DNA, which was subsequently converted into a double-stranded cDNA template. The DNA template was used to transcribe cRNA and incorporate a biotin-conjugated nucleotide. A total of $10 \mu \mathrm{g}$ of cRNA from each sample was hybridized with the mouse MG 4302.0 GeneChips (Affymetrix; Thermo Fisher Scientific, Inc.) for $16 \mathrm{~h}$ at $45^{\circ} \mathrm{C}$, with constant rotation at $3.5 \mathrm{x}$ g. Following hybridization, the microarray was washed and stained in an automated fluidics station (Affymetrix GeneChip Hybridization Wash and Stain kit; Affymetrix; Thermo Fisher Scientific, Inc.). Quality controls were assessed, which were demonstrated to be within acceptable limits for all arrays. Signals were quantified by detection of bound phycoerythrin using an Affymetrix GeneChip Scanner 3000 (Affymetrix; Thermo Fisher Scientific, Inc.) to generate CEL data. The CEL data were then subjected to analysis using DNA-Chip (dChip) software (version 2010; http://www.dchip. net/) (17) and array hybridization signal levels were normalized using the invariant set normalization method, which is part of the dChip software. 
Microarray data analysis. Microarray data were assessed and normalized to exclude background signals and analyzed using the dChip (version 2010; http://www.dchip.net/) (17) with a model-based computation. All arrays were normalized to a common baseline array and the expression levels of each gene in all samples were computed using the perfect match-mismatch differences for all probes in a probe set. A combined analysis was performed to identify the genes that exhibited different expression patterns between the experimental group and control group. For the extracted genes, hierarchical clustering was performed to visualize the changes in gene expression between the Vc- and AA-treated groups. Enrichment analysis of the differentially expressed genes was performed manually using relevant bioinformatics databases including Gene Ontology (GO; http://www.geneontology.org), GenBank (National Center for Biotechnology Information; http://www.ncbi.nlm.nih.gov/), LocusLink (http://www.ncbi. nlm.nih.gov/LocusLink), PubMed and Kyoto Encyclopedia of Genes and Genomes (KEGG; http://www.genome.ad.jp). The protein-protein interaction network was constructed using Cytoscape 3.2 software (www.genomeweb.com), which incorporates the hub genes and GO/KEGG terms. Enrichment of GO/KEGG terms by differentially expressed genes (DEGs) was assessed using the SciPy module in Python by computing a unilateral Fisher exact $\mathrm{P} \leq 0.05$.

Reverse transcription-quantitative (RT- $q) P C R$. Microarray data for a selection of genes were confirmed via RT-qPCR analysis. Total RNA $(0.8 \mu \mathrm{g})$ was extracted as aforementioned and reverse-transcribed into complementary DNA using the PrimeScript RT reagent kit (Takara Bio, Inc.) according to the manufacturer's protocol at $37^{\circ} \mathrm{C}$ for $15 \mathrm{~min}$ and $85^{\circ} \mathrm{C}$ for 5 sec. qPCR was subsequently performed using $\mathrm{SYBR}^{\circledR}$ Green PCR Master Mix (Applied Biosystems; Thermo Fisher Scientific, Inc.) following the manufacturer's protocol. The following thermocycling conditions were used: 1 cycle at $50^{\circ} \mathrm{C}$ for $2 \mathrm{~min}$ and $95^{\circ} \mathrm{C}$ for $10 \mathrm{~min}$, followed by 40 cycles at $95^{\circ} \mathrm{C}$ for $10 \mathrm{sec}$ and $60^{\circ} \mathrm{C}$ for $30 \mathrm{sec}$; a final elongation cycle at $95^{\circ} \mathrm{C}$ for $15 \mathrm{sec}, 72^{\circ} \mathrm{C}$ for $30 \mathrm{sec}$ and $95^{\circ} \mathrm{C}$ for $15 \mathrm{sec}$, using the ABI7300 Real-Time PCR System (Applied Biosystems; Thermo Fisher Scientific, Inc.) according to the manufacturer's protocol. Relative expression levels were quantified using the $2^{-\Delta \Delta C q}$ method (18) and normalized to the internal reference gene $\beta$-actin. PCR amplifications were performed in triplicate. RNA quality was confirmed by denaturing agarose gel electrophoresis, which produced two sharp and distinct bands at $18 \mathrm{~S}$ and $28 \mathrm{~S}$ and $\mathrm{OD}_{260} / \mathrm{OD}_{280}$ ratios were at a range of 1.9-2.1. The primer sequences were listed in Table SI.

If the expression level in the AA group divided by that in a control was $>1$, the gene was considered to be upregulated. Furthermore, the upward trend of the results of the chip means that the gene is upregulated. Therefore, the results of genes were all upregulated, indicating that the results of the qPCR and chip (upward trend) were consistent. The expression level of gene was $<1$ (not $<0$ ), which is consistent with the chip result (downward trend).

Statistical analysis. SPSS 11.5 software (SPSS, Inc.) was used to determine the inhibitory concentration of AA and 'Probit analysis' in SPSS software was used to determine

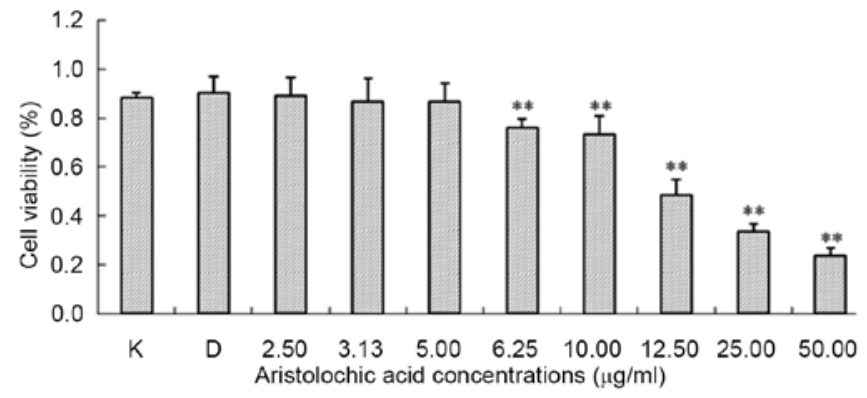

Figure 1. Effect of different aristolochic acid concentrations on ESC proliferation. ${ }^{* *} \mathrm{P}<0.05$ vs. control group. ESC, embryonic stem cell; $\mathrm{K}$, control group; D, DMSO group.

the $\mathrm{IC}_{10}$ value. Statistical analysis was performed by filtering out the genes with absent calls and genes with significantly different expression levels were identified using the following formula: $\mathrm{E} / \mathrm{B}>2$ or $\mathrm{B} / \mathrm{E}>2$, where $\mathrm{E}$ is the expression value in the experimental group and $\mathrm{B}$ is the expression value in the control group. One-way analysis of variance with Tukey's post-hoc test was performed for comparisons among groups in Fig. $1 . \mathrm{P}<0.05$ was considered to indicate a statistically significant difference.

\section{Results}

Cytotoxicity of AA on mESCs. The cytotoxicity of AA in ESCs was assessed via the MTT assay. The results demonstrated that the viability of ESCs decreased in a concentration-dependent manner following treatment with AA for $48 \mathrm{~h}$. Fig. 1 presents the effect of different concentrations of AA on the proliferation of ESCs. The $\mathrm{IC}_{10}$ was selected for the microarray experiments and for validation of microarray data via RT-qPCR analysis. The results demonstrated that ESCs were more susceptible to AA compared with non-stem cells (such as renal tubular epithelial cells) and the $\mathrm{IC}_{10}(5.20 \mu \mathrm{g} / \mathrm{ml})$ was selected for the subsequent experiments.

Changes in the gene expression profile of ESCs following treatment with $A A$. Compared with the expression levels in the control group, 72 DEGs were dysregulated (49 upregulated and 23 downregulated genes) by $>2$ fold (Table SII), 23 DEGs were dysregulated (15 upregulated and 8 downregulated genes) by $>3$ fold and five DEGs were dysregulated (4 upregulated and 1 downregulated gene) by $>4$ fold in the AA group (Fig. 2A). Among these genes, the expression of calcium binding and coiled-coil domain 2 was upregulated by 6 -fold compared with that in the control group and serine incorporator 3 was upregulated by 4.68 -fold. Tumor protein 53 apoptosis effector (Perp) was upregulated by 4.38 -fold, while suprabasin was upregulated by 4 -fold, and cation transport regulator-like 1 (E. coli) (Chac1) was downregulated by 4.17 -fold.

Clustering analysis. Clustering analysis was performed to classify different data groups into clusters according to distance measurement (the length of the line segment). The results demonstrated that the trend of gene expression within the drug-treated group was consistent and that the gene expression within the control group also presented a consistent trend, 
A

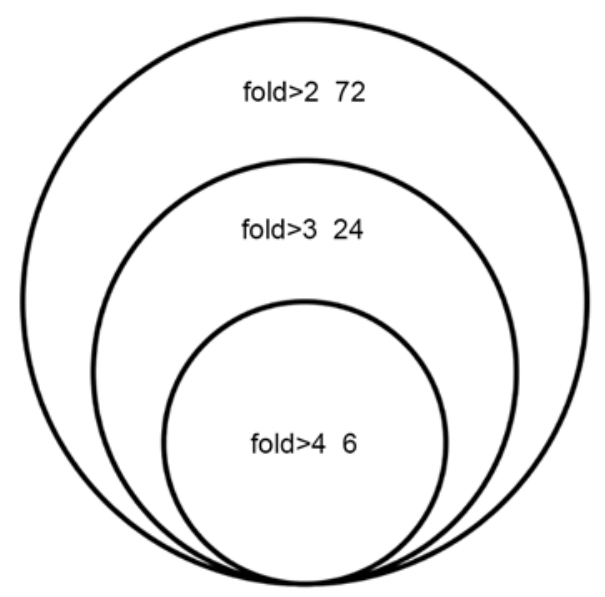

B



Figure 2. Fold changes of DEGs and clustering analysis. (A) Number of DEGs with different magnitudes of fold change. (B) Clustering analysis was used to classify different DEGs into clusters, which are displayed in a heatmap. K1-K3 referred to the different samples of the three experiments in the control group, which were arranged according to the photographic correlation. DEG, differentially expressed gene; AA, aristolochic acid.

which may be divided into the AA group or the control group (Fig. 2B). The results indicated that differential gene expression grouping of microarray data was in agreement with the experimental groups.

Bioinformatics analysis. The functional terms in the GO category biological process enriched by the DEGs are presented in Table SIII. DEGs that were changed by $>2$ fold in the AA vs. control group were selected for analysis. The downregulated DEGs were indicated to have roles in 'metabolic process', 'gluconeogenesis', 'cytolysis', 'defense response to Gram-negative bacterium', 'positive regulation of cell-substrate adhesion', 'defense response to gram-positive bacterium', 'forebrain development', 'steroid metabolic process', 'cholesterol metabolic process', 'neuron migration' and 'negative regulation of mesenchymal cell proliferation' as the top 10 biological process terms. The upregulated DEGs were enriched in the following: 'Positive regulation of intracellular signal transduction', 'negative regulation of cysteine-type endopeptidase activity involved in apoptotic process', 'positive regulation of heart rate', 'positive regulation of focal adhesion assembly', 'gluconeogenesis', 'positive regulation of smooth muscle contraction', 'positive regulation of sodium ion transport', 'intrinsic apoptotic signaling pathway by p53 class mediator', 'transmembrane transport' and 'lung development'. Furthermore, GO terms in the categories cellular component and molecular function were determined to further estimate gene functions (Table SIV). The results demonstrated that in the category cellular component, the most enriched terms were 'extracellular region', 'rough endoplasmic reticulum lumen', 'extracellular exosome', 'golgi cis cisterna', 'mitochondrion', 'microvillus', 'trans-golgi network transport vesicle', 'extracellular space', 'costamere' and 'cell surface'. The molecular function terms included 'catalytic activity', 'lysozyme activity', 'AMP binding', 'aldehyde dehydrogenase activity', 'GO:0016620', 'structural constituent of eye lens', 'calcium ion binding', 'growth factor binding', 'ATPase activity', 'coupled to transmembrane movement of substances' and 'heparin binding'.

The results of the KEGG analysis demonstrated that these genes participated in 92 different pathways. The pathways involved and changes in the number of gene accumulated in the KEGG pathways after treatment with AA are presented in Table SV. Pathway analysis demonstrated that 'salivary secretion', 'fructose and mannose metabolism', 'biosynthesis of antibiotics glycolysis/gluconeogenesis', the 'insulin signaling pathway', the 'AMP-activated protein kinase (AMPK) signaling pathway' and the 'p53 signaling pathway' were involved in early AA-induced toxicity.

Protein-protein interaction network analysis demonstrated that fructose-1, 6-bisphosphatase (Fbp)1 and Fbp2 were two important hub genes (Fig. 3). In addition, Ada, Tap1 and Abcblb were indicated to participate in primary immunodeficiency, while Perp and Pmaip1 have vital roles in the p53 signaling pathway.

Validation ofmicroarraydata via RT-qPCRanalysis.RT-qPCR analysis was performed to validate the microarray data. A total of four genes from the aforementioned experiments were selected [Perp, Chac1, adrenoceptor $\beta 2$ (Adrb2) and Wnt6]. The results demonstrated consistency between the microarray data and the RT-qPCR data (Fig. 4), indicating good reliability and reproducibility of the microarray data in the present study.

\section{Discussion}

Akebia quinata is a traditional Chinese medicinal plant that is commonly used for treating different types of diseases. Recently, one of its components, AA, has raised concerns. In the $1990 \mathrm{~s}$, several patients who consumed medicines containing AA exhibited different degrees of anemia, mild tubular proteinuria, extensive hypocellular interstitial fibrosis, tubular atrophy and even ARF. These AA-induced disorders were named AAN. The effects of AA-induced toxicity have been studied in several different animal models (19). Although conventional animal tests are able to demonstrate the toxic effects of drugs on the whole animal, most of the indexes are descriptive, lack high sensitivity and specificity, and seldom indicate the molecular mechanisms associated with toxicity. Although it is well-known that AA causes renal damage (20), the molecular mechanisms of AA-induced toxicity still remain 

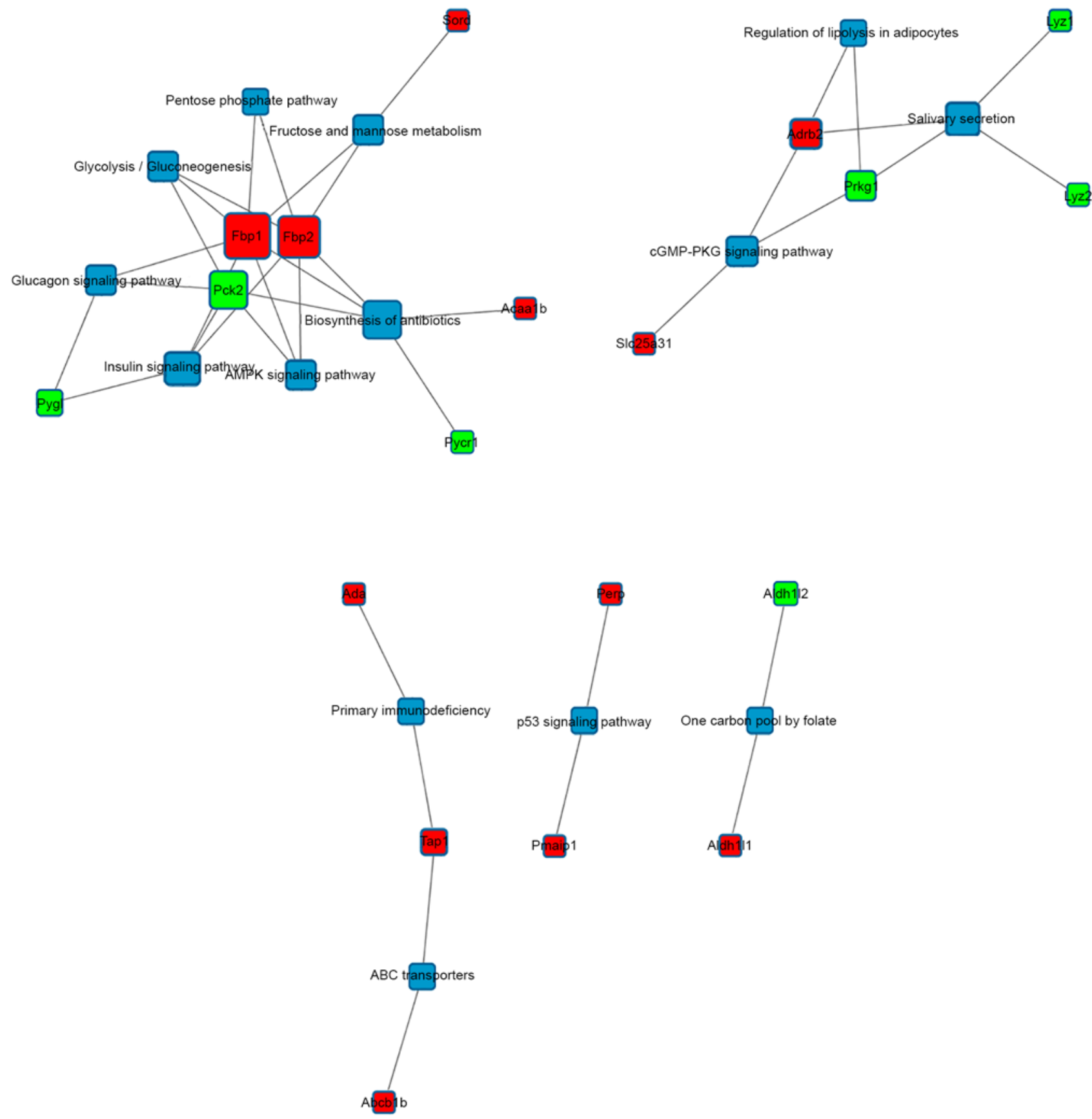

Figure 3. Network analysis for early aristolochic acid-induced toxicity. Red represents upregulation, green represents downregulation and blue is the name of the pathway.

elusive. Thus, the renal toxicity of AA requires further investigation to prevent renal damage and appropriately use this Chinese herbal medicine.

Clinical studies have indicated that AA-induced genotoxicity is a primary cause of renal tubule epithelial cell apoptosis and damage. As a tumor suppressor gene, p53 is able to regulate various cellular functions, including the cell cycle, DNA repair and apoptosis. Perp is a proapoptotic target of p53. It has been reported that p53-dependent overexpression of Perp in proximal tubular epithelium induces ischemia/reperfusion injury (21). Another study suggested that Perp may induce mitochondrial permeability to exacerbate injury in vitro (22). Lord et al (23) demonstrated that in AAN, adducts with DNA formed by AA lead to excessive tubular epithelial cell apoptosis via the p53-mediated signaling pathway. In the present study, Perp was significantly upregulated. In addition, the target genes of p53, DNA-damage-inducible transcript 4-like and transformed mouse 3T3 cell double minute, were both elevated, indicating that DNA damage and apoptosis were induced by AA and the toxic effects may be caused by activation of the 553 signaling pathway.

Fbp, a key gluconeogenic enzyme, was demonstrated to be directly suppressed by 5-aminoimidazole-4-carboxamide ribonucleoside, which is a compound for AMPK activation (24). Shi et al (25) demonstrated that Fbp1 regulates cell proliferation and glycolysis through a hypoxia-inducible factor $1 \alpha$-dependent hypoxic response in breast cancer cells. In clinical practice, pediatric patients with idiopathic 


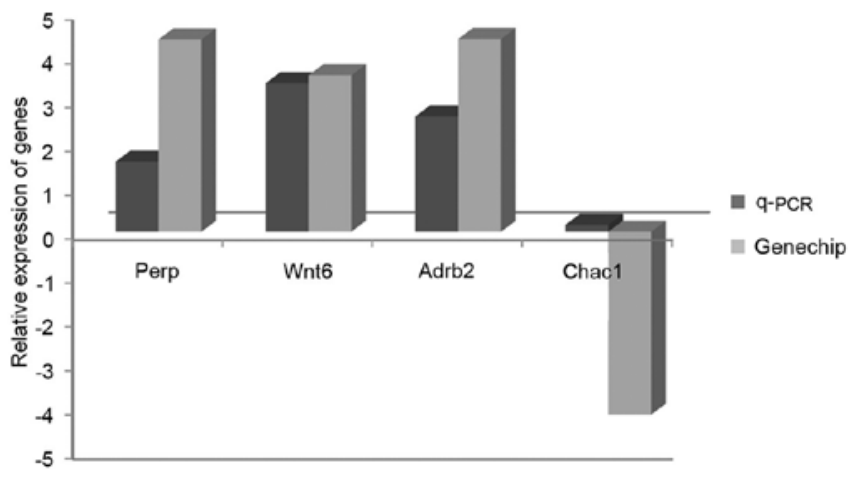

Figure 4. Validation of the microarray data by reverse transcription-qPCR of selected differentially expressed genes indicated good consistency with microarray data. The relative expression level refers to the level of gene in the AA group divided by that in a control. q-PCR, quantitative PCR; Perp, tumor protein 53 apoptosis effector; Adrb2, adrenoceptor $\beta 2$; Chac1, cation transport regulator-like 1.

nephrotic syndrome (INS) have significantly higher Fbp1 activity and protein concentrations in urine compared with healthy children, suggesting that Fbp1 activity in urine may be considered an indicator of damage to renal proximal tubules in pediatric patients with INS $(26,27)$. In the present study, elevated Fbp1 and Fbp2 levels were observed and pathway analysis demonstrated that Fbp1 and Fbp2 participated in certain metabolic pathways, including fructose and mannose metabolism, glycolysis/gluconeogenesis, biosynthesis of antibiotics and the AMPK signaling pathway.

To confirm the results of the microarray analysis, RT-qPCR was performed on four DEGs and the results obtained with the two methods regarding the changes in gene expression were compared. Gene chip is an effective tool for drug toxicity studies, and in the present study, it was used to assess the molecular mechanisms of the nephrotoxicity of AA on ESCs in vitro. The results of the present study require further analysis and validation at the genomic and proteomic levels. With the application of novel gene chip technology, the toxicity of drugs may be assessed in a more comprehensive and objective way. The present study also provides valuable information when screening for nephrotoxicity-associated genes to establish and improve the database of nephrotoxicity gene expression profiles of traditional Chinese medicines.

Genomics technology may be used to observe the damage caused by drugs in the early stage of disease or prior to changes of clinical biochemical indexes and histopathology (28). In the present study, a genomics analysis was used to evaluate the toxicology of ESCs. The GO functional enrichment analysis in the category of biological process of drug effects on ESCs was investigated after $\mathrm{AA}$ treatment at the concentration of $\mathrm{IC}_{10}$. The changes of gene expression in ESCs were investigated and nephrotoxicity markers were identified based on ESC genomics. These results may be used to evaluate the toxicity of traditional Chinese medicine drugs and provide a rapid, sensitive and reliable toxicity marker for the evaluation of nephrotoxicity of traditional Chinese medicines in the future. In the present study, AA was used at its $\mathrm{IC}_{10}$ concentration, which is more in line with the normal condition than the $\mathrm{IC}_{50}$. General toxicology experiments use $\mathrm{IC}_{50}$ or higher concentration, which is not conducive to the detection of toxic markers. As embryonic stem cells are particularly sensitive, the present study used the $\mathrm{IC}_{10}$, to detect toxicity changes in the early stage after AA administration (29). Thus, the $\mathrm{IC}_{10}$ is more conducive to the evaluation of early toxicity changes, and the $\mathrm{IC}_{10}$ was selected in the present study.

Wnt6 is considered a key gene in the development of renal tubules (30). In the present study, ESCs were used and it was attempted to investigate whether AA may have nephrotoxicity. Therefore, Wnt6 is important in AA-induced early nephrotoxicity. For Chacl, it was reported that human Chac1 protein degrades glutathione and mRNA induction is regulated by the transcription factors activating transcription factor (ATF) 4 and ATF3 and a bipartite ATF/cAMP response element regulatory element (31), indicating that Chac1 is an important gene in the ATF4-ATF3 axis. In addition, ATF3 was able to attenuate cyclosporin A-induced nephrotoxicity by downregulating C/EBP homologous protein in HK-2 cells (32). Therefore, Chacl may also be important in nephrotoxicity.

The limitation of the present study is that assessment was only performed via bioinformatics analysis. Thus, in prospective studies, it will be endeavored to perform biological experiments in vivo. In addition, although AA-induced DNA damage and apoptosis were determined in the present study, it remains elusive whether the toxic effect of AA on mESCs is exerted through the generation of AA-DNA adducts and activating the p53 pathway, which requires further investigation. Furthermore, validation experiments could not be performed. Thus, validation experiments for Fbp1 and Fbp2 in early AA toxicity are also required to be performed in future experiments.

In conclusion, the results of the present study demonstrated that Fbp1 and -2 are two important hub genes, and Perp and Pmaipl have vital roles in the p53 signaling pathway as part of the mechanisms of the nephrotoxicity of AA. Taken together, the results of the present study provide potential biomarkers for the early toxicity of AA.

\section{Acknowledgements}

Not applicable.

\section{Funding}

This study was funded by the National Basic Research Program of China (grant no. 2011CB505302) and Tianjin Health and Family Planning Commission Chinese Medicine, Integrative Medicine Research Special Project (grant no. 2017073).

\section{Availability of data and materials}

The datasets used and/or analyzed during the current study are available in the National Center for Biotechnology Information Gene Expression Omnibus, (GEO; http://www.ncbi.nlm.nih. gov/geo/; accession no. GSE162195).

\section{Authors' contributions}

LW, SSM and YHB wrote the initial draft; LW and SSM performed the data analysis; LW and YHB conceived and designed the study. All authors have seen the final draft and agreed to the contents. LW and SSM checked and approved the authenticity of the raw data. 


\section{Ethics approval and consent to participate}

This study was approved by the (animal) ethics committee of Tianjin University of Traditional Chinese Medicine (Tianjin, China).

\section{Patient consent for publication}

Not applicable.

\section{Competing interests}

The authors declare that they have no competing interests.

\section{References}

1. Yun KY, Xu ZU and Song JY: Traditional Chinese medicine containing aristolochic acids and their detection. Sci Sin Vitae 49: 238-249, 2019.

2. Kuo PC, Li YC and Wu TS: Chemical constituents and pharmacology of the aristolochia (mădōu ling) species. J Tradit Complement Med 2: 249-266, 2012.

3. Li J, Zhang L, Jiang Z, Shu B, Li F, Bao Q and Zhang L: Toxicities of aristolochic acid I and aristololactam I in cultured renal epithelial cells. Toxicol In Vitro 24: 1092-1097, 2010.

4. Honarpisheh M, Foresto-Neto O, Steiger S, Kraft F, Koehler P, von Rauchhaupt E, Potempa J, Adamowicz K, Koziel J and Lech M: Aristolochic acid I determine the phenotype and activation of macrophages in acute and chronic kidney disease. Sci Rep 8: 12169, 2018.

5. Yang L, Li X and Wang H: Possible mechanisms explaining the tendency towards interstitial fibrosis in aristolochic acid-induced acute tubular necrosis. Nephrol Dial Transplant 22: 445-456, 2007.

6. Debelle FD, Vanherweghem JL and Nortier JL: Aristolochic acid nephropathy: A worldwide problem. Kidney Int 74: 158-169, 2008.

7. Chen D, Tang Z, Luo C, Chen H and Liu Z: Clinical and pathological spectrums of aristolochic acid nephropathy. Clin Nephrol 78: 54-60, 2012

8. Bastek H, Zubel T, Stemmer K, Mangerich A, Beneke S and Dietrich DR: Comparison of aristolochic acid I derived DNA adduct levels in human renal toxicity models. Toxicology 420 29-38, 2019.

9. Fuchs TC and Hewitt P: Biomarkers for drug-induced renal damage and nephrotoxicity-an overview for applied toxicology. AAPS J 13: 615-631, 2011.

10. Mori Y, Kondo C, Tonomura Y, Torii M and Uehara T: Identification of potential genomic biomarkers for early detection of chemically induced cardiotoxicity in rats. Toxicology 271 36-44, 2010.

11. Pal R, Mamidi MK, Das AK and Bhonde R: Human embryonic stem cell proliferation and differentiation as parameters to evaluate developmental toxicity. J Cell Physiol 226: 1583-1595, 2011.

12. Ahuja YR, Vijayalakshmi V and Polasa K: Stem cell test: A practical tool in toxicogenomics. Toxicology 231: 1-10, 2007.

13. Cezar GG, Quam JA, Smith AM, Rosa GJ, Piekarczyk MS Brown JF, Gage FH and Muotri AR: Identification of small molecules from human embryonic stem cells using metabolomics. Stem Cells Dev 16: 869-882, 2007.

14. Asaba A, Okabe S, Nagasawa M, Kato M, Koshida N, Osakada T, Mogi K and Kikusui T: Developmental social environment imprints female preference for male song in mice. PLoS One 9: e87186, 2014.
15. Fagundez CB, Loresi MA, Ojea Quintana ME, Delcourt SM, Testa R, Gogorza SJ and Argibay PF: A simple approach for mouse embryonic stem cells isolation and differentiation inducing embryoid body formation. Cell Biol Int 33: 1196-1200, 2009.

16. West PR, Weir AM, Smith AM, Donley EL and Cezar GG: Predicting human developmental toxicity of pharmaceuticals using human embryonic stem cells and metabolomics. Toxicol Appl Pharmacol 247: 18-27, 2010.

17. Li C and Wing H: DNA-Chip Analyzer (dChip). The analysis of gene expression data: Methods and software. Parmigiani G, Garrett ES, Irizarry R and Zeger SL (eds). Springer, New York, pp120-141, 2003.

18. Livak KJ and Schmittgen TD: Analysis of relative gene expression data using real-time quantitative PCR and the 2(-Delta Delta C(T)) method. Methods 25: 402-408, 2001.

19. Mei N, Arlt VM, Phillips DH, Heflich RH and Chen T: DNA adduct formation and mutation induction by aristolochic acid in rat kidney and liver. Mutat Res 602: 83-91, 2006.

20. De Broe ME, Curhan GC and Forman JP: Nephropathy induced by aristolochic acid (AA) containing herbs. UpToDate, 2018.

21. Singaravelu K, Devalaraja-Narashimha K, Lastovica B and Padanilam BJ: PERP, a p53 proapoptotic target, mediates apoptotic cell death in renal ischemia. Am J Physiol Renal Physiol 296: F847-F858, 2009.

22. Singaravelu K and Padanilam B: The role of p53 pro-apoptotic target, PERP, induces mitochondrial permeability and apoptosis in hypoxic renal cells. FASEB J 22 (1 Suppl): 730.11, 2008.

23. Lord GM, Hollstein M, Arlt VM, Roufosse C, Pusey CD, Cook T and Schmeiser HH: DNA adducts and p53 mutations in a patient with aristolochic acid-associated nephropathy. Am J Kidney Dis 43: e11-e17, 2004.

24. Schimmack G, Defronzo RA and Musi N: AMP-activated protein kinase: Role in metabolism and therapeutic implications. Diabetes Obes Metab 8: 591-602, 2006

25. Shi L, He C, Li Z, Wang Z and Zhang Q: FBP1 modulates cell metabolism of breast cancer cells by inhibiting the expression of HIF-1 $\alpha$. Neoplasma 64: 535-542, 2017.

26. Kępka A, Dariusz Szajda S, Stypułkowska A, Waszkiewicz N, Jankowska A, Chojnowska S and Zwierz K: Urinary fructose-1, 6-bisphosphatase activity as a marker of the damage to the renal proximal tubules in children with idiopathic nephrotic syndrome. Clin Chem Lab Med 46: 831-835, 2008.

27. Kepka A, Szajda SD and Zwierz K: Fructose-1, 6-bisphosphatase-marker of damage to proximal renal tubules. Pol Merkur Lekarski 24: 125-130, 2008 (In Polish).

28. Upadhyaya Y, Xie L, Salama P, Cao S, Nho K, Saykin AJ and Yan J, For The Alzheimer's Disease Neuroimaging Initiative: Differential co-expression analysis reveals early stage transcriptomic decoupling in alzheimer's disease. BMC Med Genomics 13 (Suppl 5): S53, 2020.

29. Hsin YH, Cheng CH, Tzen JT, Wu MJ, Shu KH and Chen HC: Effect of aristolochic acid on intracellular calcium concentration and its links with apoptosis in renal tubular cells. Apoptosis 11: 2167-2177, 2006

30. Itäranta P, Lin Y, Peräsaari J, Roël G, Destrée O and Vainio S: Wnt-6 is expressed in the ureter bud and induces kidney tubule development in vitro. Genesis 32: 259-268, 2002.

31. Crawford RR, Prescott ET, Sylvester CF, Higdon AN, Shan J, Kilberg MS and Mungrue IN: Human CHACl protein degrades glutathione, and mRNA induction is regulated by the transcription factors ATF4 and ATF3 and a bipartite ATF/CRE regulatory element. J Biol Chem 290: 15878-15891, 2015.

32. Choi YM, Cho HY, Anwar MA, Kim HK, Kwon JW and Choi S: ATF3 attenuates cyclosporin A-induced nephrotoxicity by downregulating $\mathrm{CHOP}$ in HK-2 cells. Biochem Biophys Res Commun 448: 182-188, 2014

This work is licensed under a Creative Commons Attribution-NonCommercial-NoDerivatives 4.0 International (CC BY-NC-ND 4.0) License. 